\title{
Stochastic climate theory and modeling
}

Article

Accepted Version

Franzke, C. L. E., O'Kane, T. J., Berner, J., Williams, P. D. and Lucarini, V. (2015) Stochastic climate theory and modeling. Wiley Interdisciplinary Reviews: Climate Change, 6 (1). pp. 6378. ISSN 1757-7799 doi: https://doi.org/10.1002/wcc.318 Available at https://centaur.reading.ac.uk/38700/

It is advisable to refer to the publisher's version if you intend to cite from the work. See Guidance on citing.

To link to this article DOI: http://dx.doi.org/10.1002/wcc.318

Publisher: Wiley

All outputs in CentAUR are protected by Intellectual Property Rights law, including copyright law. Copyright and IPR is retained by the creators or other copyright holders. Terms and conditions for use of this material are defined in the End User Agreement.

\section{www.reading.ac.uk/centaur}

\section{CentAUR}

Central Archive at the University of Reading

Reading's research outputs online 


\title{
Stochastic Climate Theory and Modelling
}

\author{
Christian L. E. Franzke*1, Terence J. O'Kane ${ }^{2}$, Judith Berner ${ }^{3}$, Paul D. Williams ${ }^{4}$, and \\ Valerio Lucarini ${ }^{1,5}$ \\ ${ }^{1}$ Meteorological Institute and Centre for Earth System Research and Sustainability (CEN), \\ University of Hamburg, Hamburg, Germany \\ ${ }^{2}$ Centre for Australian Weather and Climate Research, CSIRO Marine and Atmospheric \\ Research, Hobart, Australia \\ ${ }^{3}$ National Center for Atmospheric Research, Boulder, USA \\ ${ }^{4}$ Department of Meteorology, University of Reading, Reading, UK \\ ${ }^{5}$ Department of Mathematics and Statistics, University of Reading, Reading, UK
}

August 27, 2014

\begin{abstract}
Stochastic methods are a crucial area in contemporary climate research and are increasingly being used in comprehensive weather and climate prediction models as well as reduced order climate models. Stochastic methods are used as subgrid-scale parameterizations as well as for model error representation, uncertainty quantification, data assimilation and ensemble prediction. The need to use stochastic approaches in weather and climate models arises because we still cannot resolve all necessary processes and scales in comprehensive numerical weather and climate prediction models. In many practical applications one is mainly interested in the largest and potentially predictable scales and not necessarily in the small and fast scales. For instance, reduced order models can simulate and predict large scale modes. Statistical mechanics and dynamical systems theory suggest that in reduced order models the impact of unresolved degrees of freedom can be represented by suitable combinations of deterministic and stochastic components and non-Markovian (memory) terms. Stochastic approaches in numerical weather and climate prediction models also lead to the reduction of model biases. Hence, there is a clear need for systematic stochastic approaches in weather and climate modelling. In this review we present evidence for stochastic effects in laboratory experiments. Then we provide an overview of stochastic climate theory from an applied mathematics perspectives. We also survey the current use of stochastic methods in comprehensive weather and climate prediction models and show that stochastic parameterizations have the potential to remedy many of the current biases in these comprehensive models.
\end{abstract}

\footnotetext{
${ }^{*}$ Corresponding Author; Meteorological Institute and Centre for Earth System Research and Sustainability, University of Hamburg, Grindelberg 7, D-20144 Hamburg, Germany; Email: christian.franzke@uni-hamburg.de
} 


\section{Introduction}

The last few decades have seen a considerable increase in computing power which allows the simulation of numerical weather and climate prediction models with ever higher resolution and the inclusion of ever more physical processes and climate components (e.g. cryosphere, chemistry). Despite this increase in computer power many important physical processes (e.g. tropical convection, gravity wave drag, clouds) are still not or only partially resolved in these numerical models. Despite the projected exponential increase in computer power these processes will not be explicitly resolved in numerical weather and climate models in the foreseeable future ${ }^{120,171}$. For instance, Dawson et al. ${ }^{24}$ have demonstrated using the ECMWF integrated forecast system that extremely high resolutions (T1279, which corresponds to a grid spacing of about $16 \mathrm{~km}$ ) are required to accurately simulate the observed Northern hemispheric circulation regime structure. This resolution, typical for limited area weather and climate models used for short term prediction, remains unfeasible for the current generation of high resolution global climate models due to computational and data storage requirements. Hence, these missing processes need to be parameterized, i.e. they need to be represented in terms of resolved processes and scales ${ }^{153}$. This representation is important because small-scale (unresolved) features can impact the larger (resolved) scales ${ }^{84,162}$ and lead to error growth, uncertainty and biases.

At present, these parameterizations are typically deterministic, relating the resolved state of the model to a unique tendency representing the integrated effect of the unresolved processes. These "bulk parameterizations" are based on the notion that the properties of the unresolved subgrid-scales are determined by the large-scales. However, studies have shown that resolved states are associated with many possible unresolved states ${ }^{22,144,167}$. This calls for stochastic methods for numerical weather and climate prediction which potentially allow a proper representation of the uncertainties, a reduction of systematic biases and improved representation of long-term climate variability. Furthermore, while current deterministic parameterization schemes are inconsistent with the observed power-law scaling of the energy spectrum ${ }^{5,142}$ new statistical dynamical approaches that are underpinned by exact stochastic model representations have emerged that overcome this limitation. The observed power spectrum structure is caused by cascade processes. Recent theoretical studies suggest that these cascade processes can be best represented by a stochastic non-Markovian Ansatz. Non-Markovian terms are necessary to model memory effects due to model reduction ${ }^{19}$. It means that in order to make skillful predictions the model has to take into account also past states and not only the current state (as for a Markov process).

We first review observational evidence of stochasticity in laboratory geophysical fluid experiments (section 2 ), then discuss stochastic climate theory in fast-slow systems (system 3). In section 4 we present statistical physics approaches and in section 5 we review the current state of stochastic-dynamic weather and climate modelling. We close with an outlook and challenges for the future of weather and climate modelling (section 6 ).

\section{Laboratory Evidence of Stochasticity}

Research on the climate system is somewhat hindered by the obvious difficulties of performing reproducible experiments on the atmosphere and ocean in different parameter regimes. For example, an optical physicist studying the nonlinear response of isolated atoms to intense electromagnetic waves can easily change the incident wavelength $^{110}$. In contrast, climate scientists cannot (and arguably should not!) change the rotation rate of the planet or the intensity of the incoming solar radiation. To some extent, numerical simulations come to the rescue, by allowing us to perform virtual experiments. However, the grid spacing in climate models is orders of magnitude larger than the smallest energized scales in the atmosphere and ocean, introducing biases.

Fortunately, there is another option available to us. It is possible to exploit dynamical similarity ${ }^{30}$ to study analogues of planetary fluid flow in bespoke laboratory experiments. The traditional set-up is the classic rotating annulus, which has been used for decades to study baroclinic instability and other large-scale phenomena ${ }^{61}$. Recent observations of small-scale inertia-gravity waves embedded within a large-scale baroclinic wave ${ }^{85,172,173}$ have allowed the scale interactions between these two modes to be studied in a laboratory fluid for the first 
time. The experimental apparatus consists of a two-layer isothermal annulus forced by a differentially rotating lid, which drives a shear across the internal interface and represents the mid-latitude tropospheric wind shear.

The large-scale baroclinic wave in these laboratory experiments exhibits regime behavior, equilibrating at finite amplitude with a zonal wavenumber of typically 1, 2, or 3 . These simple wave modes are regarded as prototypes of the more complicated regime behavior in the atmosphere, such as mid-latitude blocking ${ }^{160,164}$. A notable finding from repeated experiments using this apparatus is that small-scale inertia-gravity waves can induce large-scale regime transitions, despite the separation of wavelengths by an order of magnitude ${ }^{168}$. An example of this process is illustrated in Figure 1. A wavenumber 2 mode without co-existing inertia-gravity waves (upper row) remains a wavenumber 2 mode indefinitely, drifting around the annulus with the zonal-mean flow. In contrast, with the same parameter values, a wavenumber 2 mode with co-existing inertia-gravity waves (lower row) is found to have a finite probability of transitioning to a wavenumber 1 mode. The amplitude of the inertia-gravity waves is controlled here without directly affecting the large-scale mode, by slightly varying the interfacial surface tension between the two immiscible fluid layers.

The laboratory transitions discussed above are reminiscent of noise-induced transitions between different equilibrium states in a meta-stable dynamical system ${ }^{158}$. To test this interpretation, a quasi-geostrophic numerical model that captures the meta-stability of the large-scale flow in the rotating annulus ${ }^{174}$ was run with and without weak stochastic forcing added to the potential vorticity evolution equation for each fluid layer. The stochastic forcing was an approximate representation of the inertia-gravity waves, which are inherently ageostrophic and are therefore forbidden from the quasi-geostrophic model. Consistent with the laboratory experiments, only when the noise term was activated did the numerical simulations exhibit large-scale wave transitions in the equilibrated flow ${ }^{169}$. In further numerical experiments, the noise was found to be able to influence wavenumber selection during the developing baroclinic instability.

In summary, the above laboratory experiments constitute the first evidence in a real fluid that small-scale waves may trigger large-scale regime transitions. In a numerical model in which the small-scale waves were absent, the transitions were captured through the addition of stochastic noise. Note that the small-scale waves satisfy the dispersion relation for inertia-gravity waves and are therefore coherent in space and time, and yet apparently they are 'sensed' by the large-scale flow as if they were random fluctuations. These results have led to a possible interpretation of sudden stratospheric warmings as noise-induced transitions ${ }^{9}$. Furthermore, these laboratory results help to motivate the development of stochastic parameterizations in climate models and a more general development of stochastic climate theory.

\section{Stochastic Climate Theory}

Climate is a multi-scale system in which different physical processes act on different temporal and spatial scales ${ }^{69}$. For instance, on the micro-scale are turbulent eddies with time scales of seconds to minutes, on the meso-scale is convection with time scales of hours to days, on the synoptic scale are mid-latitude weather systems and blocking with time scales from days to weeks, on the large-scale are Rossby waves and teleconnection patterns with time scales of weeks to seasons. And there is the coupled atmosphere-ocean system with time scales of seasons to decades. The crucial point here is that all these processes acting on widely different temporal and spatial scales, interact with each other due to the inherent nonlinearity of the climate system. We have shown an illustrative laboratory example for this in the previous section.

For many practical applications we are only interested in the processes on a particular scale and not in the detailed evolution of the processes at smaller scales. Often the scales of interest are linked to inherently predictable processes, while the smaller scales processes are unpredictable. For instance, in the above laboratory experiment we are interested in the regime behavior and not in the detailed evolution of the inertia-gravity waves. In numerical simulations the fastest scales, which are typically also the smallest scales, use up the bulk of computing time, slowing down the computation of the processes of actual interest. In numerical weather and climate prediction many of the small scale processes are currently not explicitly resolved and won't be in the 
foreseeable future. This neglect of these processes can lead to biases in the simulations. Because of that the unresolved processes need to be parameterized as demonstrated in the previous section.

Stochastic climate theory is based on the concept of scale separation in space or time. Hasselmann ${ }^{56}$ was the first to propose to split the state vector $\vec{z}$ into slow climate components $\vec{x}$ and fast weather fluctuations $\vec{y}$ and then to derive an effective equation for the slow climate variables only. In this equation the effect of the now unresolved variables is partially represented as a noise term. The physical intuition behind this idea is, for example, that the aggregated effect of 'fast' weather fluctuations drives fluctuations in the 'slower' ocean circulation. To first order such a model can explain the 'red' spectrum of oceanic variables ${ }^{36,64}$. It has to be noted that there is no scale separation in the climate system. This lack of time-scale separation introduces non-Markovian (memory) effects and complicates the derivation of systematic parameterizations.

Rigorous mathematical derivations for this approach have been provided by Gottwald and Melbourne ${ }^{52}$, Khasminsky $^{66}$, Kurtz ${ }^{76}$, Melbourne and Stuart ${ }^{101}$, Papanicolaou ${ }^{124}$, Pavliotis and Stuart ${ }^{125}$. For accessible reviews see Givon et al. ${ }^{50}$ and the text book by Pavliotis and Stuart ${ }^{125}$. This approach has been applied to climate models by Majda and coworkers ${ }^{29,37,38,89-94,96}$. Climate models have the following general functional form

$$
d \vec{z}=(\tilde{F}+\tilde{L} \vec{z}+\tilde{B}(\vec{z}, \vec{z})) d t
$$

where $\tilde{F}$ denotes an external forcing, $\tilde{L}$ a linear operator and $\tilde{B}$ a quadratic nonlinear operator. Eq. (1) constitutes the form of the dynamical cores of weather and climate prediction models.

Now splitting the state vector $\vec{z}$ into slow $\vec{x}$ and fast $\vec{y}$ components (which amounts to assuming a time scale separation) and assuming that the nonlinear self-interaction of the fast modes $\tilde{B}(\vec{y}, \vec{y})$ can be represented by a stochastic process ${ }^{37,38,89,90}$ leads to a stochastic differential equation. The stochastic mode reduction approach $^{37,38,89,90}$ then predicts the functional form of reduced climate models for the slow variable $\vec{x}$ alone:

$$
d \vec{x}=(F+L \vec{x}+B(\vec{x}, \vec{x})+M(\vec{x}, \vec{x}, \vec{x})) d t+\sigma_{A} d \vec{W}_{A}+\sigma_{A}(\vec{x}) d \vec{W}_{M}
$$

Structurally new terms are a deterministic cubic term which acts predominantly as nonlinear damping and both additive and multiplicative (state-dependent) noise terms. The fundamentals of stochastic processes and calculus are explained in Box 1. The multiplicative noise and the cubic term stem from the nonlinear interaction between the resolved and unresolved modes ${ }^{37}$.

The above systematic procedure allows also a physical interpretation of the new deterministic and stochastic terms $^{37}$. The additive noise stems both from the nonlinear interaction amongst the unresolved modes and the linear interaction between resolved and unresolved modes ${ }^{37}$. 


\section{BOX 1 \\ STOCHASTIC PROCESSES}

In contrast to deterministic processes stochastic processes have a random component. See the books by Lemons $^{86}$ and Gardiner ${ }^{49}$ for intuitive introductions to stochastic processes. Typically stochastic processes are driven by white noise. White noise is a serially uncorrelated time series with zero mean and finite variance $^{49}$.

A stochastic differential equation (SDE) is a combination of a deterministic differential equation and a stochastic process. In contrast to regular calculus, stochastic calculus is not unique; i.e. different discretizations of its integral representation lead to different results even for the same noise realization. The two most important calculi are Ito and Stratonovich. See details in Gardiner ${ }^{49}$. The physical difference is that Ito calculus has uncorrelated noise forcing while Stratonovich allows for finite correlations between noise increments. Hence, physical systems have to be typically approximated by Stratonovich SDEs. On the other hand, it is mathematically straightforward to switch between the two calculi. So one only needs to make a decision at the beginning which calculus is more appropriate for modeling the system under consideration and can then switch to the mathematically more convenient form.

SDEs describe systems in a path wise fashion. The Fokker-Planck equation (FPE) describes how the probability distribution evolves over time ${ }^{49}$. Thus, SDEs and the FPE offer two different ways at looking at the same system. The parameters of SDEs and their corresponding FPE are linked; thus, one can use the FPE to estimate the parameters of the corresponding $\mathrm{SDE}^{3,146}$.

Multiplicative (or state-dependent) noise is important for deviations from Gaussianity and thus extremes. The intuition behind multiplicative noise is as follows: On a windless day the fluctuations are very small, whereas on a windy day not only is the mean wind strong but also the fluctuations around this mean are large; thus, the magnitude of the fluctuations dependent on the state of the system.

The first practical attempts at stochastic climate modelling were made using Linear Inverse Models (LIM)

$127,128,166,176$ and dynamically based linear models with additive white noise forcing ${ }^{26,27,34,35,179}$. These approaches linearise the dynamics and then add white noise and damping ${ }^{166}$ in order to make the models numerically stable (i.e. the resulting linear operator should only have negative eigenvalues to ensure stability and reliasability of the solutions). While these models have encouraging predictive skill, especially for ENSO, they can only produce Gaussian statistics and, thus, are less useful for predictions of high impact weather.

Recently there are encouraging attempts in fitting nonlinear stochastic models to data. These include multilevel regression ${ }^{70,74}$, fitting the parameters via the Fokker-Planck equation ${ }^{3,146}$, stochastic averaging ${ }^{23,103}$, optimal prediction $^{18,154}$ or Markov Chains ${ }^{21}$. Most of the previous approaches fitted the parameters of the stochastic models without taking account of physical constraints, e.g. global stability. Many studies linearized the dynamics and then added additional damping to obtain numerically stable models ${ }^{1,2,166,179}$. Majda et al. ${ }^{96}$ developed the nonlinear normal form of stochastic climate models and also physical constraints for parameter estimation. Recent studies use these physical constraints to successfully derive physically consistent stochastic climate models ${ }^{57,97,126}$.

Most of the above approaches are based on an implicit assumption of time scale separation. However, the climate system has a spectrum with no clear gaps which would provide the basis of scale separation and the derivation of reduced order models. Such a lack of time scale separation introduces memory effects into the truncated description. Memory effects mean that the equations become non-Markovian and that also past states need to be used in order to predict the next state. This can be explained by considering the interaction between a large-scale Rossby wave with a smaller scale synoptic wave. At some location the Rossby wave will favor the development of the synoptic wave. Initially this synoptic wave grows over some days without affecting the Rossby wave. Once the synoptic wave has reached a sufficient large amplitude it will start affecting the Rossby wave. Now in a reduced order model only resolving the Rossby wave but not the synoptic wave this interaction cannot be explicitly represented. However, because the Rossby wave initially triggered the synoptic wave which then in turn affects it some days later, this can be modeled with memory terms which takes into account that 
the Rossby wave has triggered at time $t_{0}$ an anomaly which will affect it at some later time $t_{n}$.

Recently, Wouters and Lucarini ${ }^{177}$ have proposed to treat comprehensively the problem of model reduction in multi-scale systems by adapting the Ruelle response theory ${ }^{136,137}$ for studying the effect of the coupling between the fast and slow degrees of freedom of the system. This theory has previously been used in a geophysical context to study the linear and nonlinear response to perturbations ${ }^{87,88}$, which also allows climate change predictions. This approach is based on the chaotic hypothesis ${ }^{48}$ and allows the general derivation of the reduced dynamics of the slow variables able to mimic the effect of the fast variables in terms of matching the changes in the expectation values of the observables of the slow variables. The ensuing parametrization includes a deterministic correction, which is a mean field result and corresponds to linear response, a general correlated noise and a non-Markovian (memory) term. These results generalize Eq. (2). In the limit of infinite time-scale separation, the classical results of the averaging method is recovered. Quite reassuringly, the same parametrizations can be found using a classical Mori-Zwanzig approach ${ }^{19}$, which is based on projecting the full dynamics on the slow variables and general mathematical results provide evidence that deterministic, stochastic and non-Markovian components should constitute the backbone of parameterizations ${ }^{17,178}$. Recent studies show improvements over approaches based on time-scale separation ${ }^{17,177,178}$.

Recent studies have shown that stochastic approaches are also important for the prediction of extreme events and tipping points ${ }^{40,41,155,156}$. Sura ${ }^{156}$ discusses a stochastic theory of extreme events. He especially focuses on deviations from a Gaussian distribution; i.e. skewness and kurtosis, as first measures of extremes. He shows that multiplicative noise plays a significant role in causing non-Gaussian distributions. Franzke ${ }^{40}$ shows that both deterministic nonlinearity and multiplicative noise are important in predicting of extreme events.

\section{Statistical Physics Approaches to Stochastic Climate Theory}

Significant progress has been achieved in the development of tractable and accurate statistical dynamical closures for general inhomogeneous turbulent flows that are underpinned by exact stochastic models (see Box 2). For an accessible review see the text book by Heinz ${ }^{58}$. The statistical dynamical closure theory, pioneered by Kraichnan ${ }^{71}$, has been recognized as a natural framework for a systematic approach to modelling turbulent geophysical flows. Closure theories like the Direct Interaction Approximation (DIA), ${ }^{71}$ for homogeneous turbulence and the Quasi-Diagonal Direct Interaction Approximation (QDIA), ${ }^{42}$ for the interaction of mean flows with inhomogeneous turbulence have exact generalized Langevin model representations ${ }^{60}$. This means that such closures are realizable; i.e. they have non-negative energy.

The first major application of turbulence closures has been the examination of the predictability of geophysical flows. Early approaches applied homogeneous turbulence models to predicting error growth ${ }^{77,79,83}$ whereas more recent advances by Frederiksen and O'Kane ${ }^{46}$, O'Kane and Frederiksen ${ }^{113}$, building on the pioneering studies of Epstein ${ }^{32}$ and Pitcher ${ }^{130}$, have enabled predictability studies of inhomogeneous strongly non-Gaussian flows typical of the mid-latitude atmosphere. Turbulence closures have also been used for SubgridScale Parameterisation (SSP) of the unresolved scales, for example eddies in atmospheric and ocean general circulation models. Since it is generally only possible to represent the statistical effects of unresolved eddies while their phase relationships with the resolved scales are lost ${ }^{100}$, statistical dynamical turbulence closures are sufficient to allow SSPs to be formulated in a completely transparent way $42,43,73,77,80,112,134$. Insights gained through the development of inhomogeneous turbulence closure theory have motivated the recent development of general stochastic forms for subgrid-scale parameterisations for geophysical flows ${ }^{68}$. 


\section{BOX 2 CLOSURE PROBLEM}

In order to describe the statistical behavior of a turbulent flow the underlying nonlinear dynamical equations must be averaged. For simplicity we consider a generic equation of motion with quadratic nonlinearity for homogeneous turbulence, in which the mean field is zero, and the fluctuating part of the vorticity in Fourier space, $\hat{\zeta_{\mathbf{k}}}$, satisfies the equation:

$$
\frac{\partial}{\partial t} \hat{\zeta}_{\mathbf{k}}(t)=K_{\mathbf{k p q}} \hat{\zeta}_{-\mathbf{p}}(t) \hat{\zeta}_{-\mathbf{q}}(t) .
$$

where $\mathbf{p}$ and $\mathbf{q}$ are the other wave numbers describing triad interactions i.e. $\mathbf{k}=\left(k_{x}, k_{y}\right)$ where $\delta(\mathbf{k}+\mathbf{p}+\mathbf{q})=1$ if $\mathbf{k}+\mathbf{p}+\mathbf{q}=0$ and 0 otherwise. Here $K_{\mathbf{k p q}}$ are the interaction or mode coupling coefficients. The correlation between the eddies can now be represented by an equation for the covariance (cumulant in terms of wavenumbers $\mathbf{k}$ and $\mathbf{l}$ ) which is found to depend on the third order cumulant in Fourier space:

$$
\frac{\partial}{\partial t}\left\langle\hat{\zeta}_{\mathbf{k}}(t) \hat{\zeta}_{-\mathbf{l}}\left(t^{\prime}\right)\right\rangle=K_{\mathbf{k p q}}\left\langle\hat{\zeta}_{-\mathbf{p}}(t) \hat{\zeta}_{-\mathbf{q}}(t) \hat{\zeta}_{-\mathbf{l}}\left(t^{\prime}\right)\right\rangle .
$$

Similarly the third order cumulant depends on the fourth order and so on such that we see that an infinite hierarchy of moment or cumulant equations is produced. Statistical turbulence theory is principally concerned with the methods by which this moment hierarchy is closed and the subsequent dynamics of the closure equations. The fact that for homogeneous turbulence the covariance matrix is diagonal greatly simplifies the problem. The majority of closure schemes are derived using perturbation expansions of the nonlinear terms in the primitive dynamical equations. The most successful theories use formal renormalization techniques ${ }^{19,58}$.

The development of renormalized turbulence closures was pioneered by Kraichnan's Eulerian DIA ${ }^{71}$ for can be readily regularised to include approximations to the indirect interactions ${ }^{45,111}$ which are required to obtain the correct inertial range scaling laws. Other homogeneous closures such as Herring's self consistent field theory ( $\mathrm{SCFT}^{59}$ ) and McComb's local energy transfer theory (LET), ${ }^{99}$ were independently developed soon after. The DIA, SCFT and LET theories have since been shown to form a class of homogeneous closures that differ only in whether and how a fluctuation dissipation theorem (FDT i.e. the linear response of a system to an infinitesimal perturbation as it relaxes toward equilibrium $)^{15,25,44,71}$ is applied. As noted earlier, a consequence of the DIA having an exact stochastic model representation is that it is physically realizable, ensuring positive energy spectra. This is in contrast with closures based on the quasi-normal hypothesis which require further modifications in order to ensure realizability; an example of such a closure is the eddy damped quasi-normal Markovian (EDQNM) closure $77,109,116$ developed as a bets Markovian fit to the DIA. The EDQNM is dependent on a choice of an eddy-damping parameter which can be tuned to match the phenomenology of the inertial range. This Markovian assumption assumes that the rate at which the memory integral decays is significantly faster than the time scale on which the covariances evolve. The relative success of these turbulence closures has enabled the further study of the statistics of the predictability of homogeneous turbulent flows ${ }^{77-79,102}$.

Frederiksen ${ }^{42}$ formulated a computationally tractable non-Markovian (memory effects) closure, the quasidiagonal direct interaction approximation (QDIA), for the interaction of general mean and fluctuating flow components with inhomogeneous turbulence and topography. The QDIA assumes that, prior to renormalisation, a perturbative expansion of the covariances are diagonal at zeroth order. In general, very good agreement has been found between the QDIA closure results and the statistics of DNS ${ }^{45,46,111}$.

The non-Markovian closures discussed above are systems of integro-differential equations with potentially long time-history integrals posing considerable computational challenges, however various ways to overcome 
these challenges exist $44,46,111-115,135$ and have been generalised to allow computationally tractable closure models for inhomogeneous turbulent flow over topography to be developed ${ }^{42,46,111}$. An alternative derivation of a stochastic model of the Navier-Stokes equations has been put forward by Memin ${ }^{107}$. It is based on a decomposition of the velocity fields into a differentiable drift and a stochastic component.

\subsection{Statistical dynamical and stochastic subgrid modelling}

Many subgrid-scale stress models assume the small scales to be close to isotropic and in equilibrium such that energy production and dissipation are in balance, similar to the eddy viscosity assumption of the Smagorinsky model $^{148}$. Using the DIA, Kraichnan ${ }^{71}$ showed that for isotropic turbulence the inertial transfer of energy could be represented as a combination of both an eddy viscous (on average energy drain from retained to subgrid scales) and stochastic back-scatter (positive semi-definite energy input from subgrid to retained scales) terms. The nonlinear transfer terms represented by eddy viscosity and stochastic back-scatter are the subgrid processes associated with the respective eddy-damping and nonlinear noise terms that constitute the right hand side of the DIA tendency equation for the two-point cumulant $\frac{\partial}{\partial t}\left\langle\hat{\zeta}_{\mathbf{k}}(t) \hat{\zeta}_{-\mathbf{k}}\left(t^{\prime}\right)\right\rangle$. Leith ${ }^{77}$ used the EDQNM closure to calculate an eddy dissipation function that would preserve a stationary $k^{-3}$ kinetic energy spectrum for twodimensional turbulence. Kraichnan ${ }^{73}$ developed the theory of eddy viscosity in two and three dimensions and was the first to identify the existence of a strong cusp in the spectral eddy viscosity near the cutoff wavenumber representing local interactions between modes below and near the cusp. Rose ${ }^{134}$ argued for the importance of eddy noise in subgrid modelling.

O'Kane and Frederiksen ${ }^{112}$ calculated QDIA based ssPs considering observed atmospheric flows over global topography and quantifying the relative importance of the subgrid-scale eddy-topographic, eddy-mean field, quadratic mean and mean field-topographic terms. They also compared the QDIA based ssPs to heuristic approaches based on maximum entropy, used to improve systematic deficiencies in ocean climate models ${ }^{62}$. While closure models may be the natural starting place for developing subgrid-scale parameterisations, their complexity makes them difficult to formulate and apply to multi-field models like GCMs, even though sucessfull studies exist ${ }^{68,180}$.

\section{Stochastic Parametrisation Schemes in Comprehensive Models}

Climate and weather predictions are only feasible because the governing equations of motion and thermodynamics are known. To solve these equations we need to resort to numerical simulations that discretize the continuous equations onto a finite grid and parameterize all processes that cannot be explicitly resolved. Such models can be characterized in terms of their dynamical core, which describe the resolved scales of motion, and the physical parameterizations, which provide estimates of the grid-scale effect of processes which cannot be resolved by the dynamical core. This general approach has been hugely successful in that nowadays predictions of weather and climate are made routinely. On the other hand, exactly through these predictions it has become apparent that uncertainty estimates produced by current state-of the art models still have shortcomings.

One shortcoming is that many physical parameterizations are based on bulk formula which are based on the assumption that the subgrid-scale state is in equilibrium with the resolved state ${ }^{118}$. Model errors might arise from a misrepresentation of unresolved subgrid-scale processes which can affect not only the variability, but also the mean error of a model ${ }^{129,141}$. An example in a comprehensive climate model is e.g., the bias in the $500 \mathrm{hPa}$ geopotential height pattern, which is reduced when the representation of the subgrid-state is refined $^{7}$ (Fig. 2 ).

In recent years, methods for the estimation of flow-dependent uncertainty in predictions have become an important topic. Ideally, uncertainties should be estimated within the physical parameterizations and uncertainty representations should be developed alongside the model. Many of these methods are "ad hoc" and added a posteriori to an already tuned model. Only first steps to develop uncertainty estimates from within the parameterizations have been attempted ${ }^{20,131}$. 
The representation of model-error in weather and climate models falls in one of two major categories: Multi-model approaches and stochastic parameterizations. In the multi-model approach each ensemble member consists of an altogether different model. The models can differ in the dynamical core and the physical parameterizations ${ }^{55,63,75}$ or use the same dynamical core but utilize either different static parameters in their physical parameterizations ${ }^{151}$ or altogether different physics packages $6,31,106,152$. Both approaches have been successful in improving predictions of weather and climate over a range of scales, as well as their uncertainty. Multi-model ensembles provide more reliable seasonal forecasts ${ }^{122}$ and are commonly used for the uncertainty assessment of climate change predictions e.g., as in the Assessment Report 5 of the Intergovernmental Panel on Climate Change (IPCC) ${ }^{157}$. Stochastic parameterizations are routinely used to improve the reliability of weather forecasts in the short- ${ }^{6}$ and medium-range ${ }^{5,10,123}$ as well as for seasonal predictions ${ }^{4,28,165}$.

In the stochastic approach, the effect of uncertainties due to the finite truncation of the model are treated as independent realizations of stochastic processes that describe these truncation uncertainties. This treatment goes back to the idea of stochastic-dynamic prediction ${ }^{33,118,130}$. While the verdict is still open if subgrid-scale fluctuations must be included explicitly via a stochastic term, or if it is sufficient to include their mean influence by improved deterministic physics parameterizations, one advantage of stochastically perturbed models is that all ensemble members have the same climatology and model bias; while for multi-parameter, multi-parameterization and multi-model ensembles each ensemble member is de facto a different model with its own dynamical attractor. For operational centers the maintenance of different parameterizations requires additional resources and due to the different biases makes post-processing very difficult.

\subsection{Stochastic Parameterizations in Numerical Weather Prediction}

Due to the chaotic nature of the dynamical equations governing the evolution of weather, forecasts are sensitive to the initial condition limiting the intrinsic predictability of the weather system ${ }^{82,84}$. Probabilistic forecasts are performed by running ensemble systems, where each member is initialized from a different initial state and much effort has gone into the optimal initialization of such ensemble systems ${ }^{63,108,161}$. Nevertheless state-ofthe-art numerical weather predictions systems continue to produce unreliable and over-confident forecasts ${ }^{14}$. Consequently, the other source of forecast uncertainty - model-error - has received increasing attention ${ }^{117,118}$. Since for chaotic systems model-error and initial condition error will both result in trajectories that will diverge from the truth, it is very difficult to disentangle them ${ }^{149}$.

The first stochastic parameterization used in an operational numerical weather prediction model was the stochastically perturbed physics tendency scheme (SPPT), sometimes also referred to as stochastic diabatic tendency or Buizza-Miller-Palmer (BMP) scheme ${ }^{13}$. SPPT is based on the notion that - especially as the horizontal resolution increases - the equilibrium assumption no longer holds and the subgrid-scale state should be sampled rather than represented by the equilibrium mean. Consequently, SPPT multiplies the accumulated physical tendencies of temperature, wind and moisture at each grid-point and time step with a random pattern that has spatial and temporal correlations. In other words, SPPT assumes that parameterization uncertainty can be expressed as a multiplicative noise term. Ensemble systems perturbed with the SPPT scheme show increased probabilistic skill mostly due to increased spread in short and medium-range ensemble forecasts ${ }^{8,13,132,159}$.

A second successful stochastic parameterization scheme, is the so-called stochastic kinetic energy-backscatter scheme (SKEBS) whose origin lies in Large-Eddy Simulation modeling ${ }^{98}$ and has recently been extended to weather and climate scales ${ }^{142,143}$. The key idea is that energy associated with subgrid processes is injected back onto the grid using a stochastic pattern generator. This method has been successfully used in a number of operational and research forecasts across a range of scales $5,6,8,10,11,16,138$. Similar to the SPPT scheme, ensemble systems with SKEBS increase probabilistic skill by increasing spread and decreasing the root-meansquare (RMS) error of the ensemble mean forecast. First results of these schemes at a convection-permitting resolution of around $4 \mathrm{~km}$ report also a positive impact on forecast skill, in particular more reliable precipitation forecasts $^{12,133}$. 


\subsection{Stochastic Parameterizations in Climate Models}

The use of stochastic parameterization in climate models is still in its infancy. Climate prediction uncertainty assessments, e.g., IPCC ${ }^{150}$, are almost exclusively based on multi-models, mostly from different research centers. Part of the problem is that on climate timescales, limited data for verification exists. A second reason is that on longer timescales, bias is a major source of uncertainty and traditional multi-models are very efficient at sampling biases, although such an experiment is poorly designed for an objective and reliable uncertainty assessment.

However, in recent years first studies have emerged which demonstrate the ability of stochastic parameterizations to reduce longstanding biases and improve climate variability (see Fig. 2 for an example). Jin and Neelin ${ }^{81}$ developed a stochastic convective parameterization that includes a random contribution to the convective available potential energy (CAPE) in the deep convective scheme. They find that adding convective noise results in enhanced eastward propagating, low-wavenumber low-frequency variability. Berner et al. ${ }^{7}$ investigate the impact of SKEBS on systematic model-error and report an improvement in the representation of convectively-coupled waves leading to a reduction in the tropical precipitation bias. Furthermore, Majda and colleagues developed systematic stochastic multi-cloud parameterizations for organized convection ${ }^{47,67,94,95}$. The multi-cloud approach is based on the assumption that organized convection involves three types of clouds and the evolution from one cloud type to another can be described by a transition matrix.

A longstanding systematic error of climate models is the underestimation of the occurrence of Northern Hemispheric blocking. Stochastic parameterizations have been demonstrated to be one way to increase their frequency ${ }^{4,28,53,165}$, although, e.g. increasing horizontal resolution, leads to similar improvements ${ }^{7,24}$. This suggests that while it might be necessary to include subgrid-scale variability in some form, the details of this representation might not matter. On the other side, Berner et al. ${ }^{7}$ argue that this degeneracy of response to different subgrid-scale forcings warrants a cautionary note: namely that a decrease in systematic error might not necessarily occur for the right dynamical reasons. The opposite holds true, as well: Due to the necessary tuning of parameters in the parameterizations of comprehensive climate models, an improvement in the formulation of a physical process might not immediately lead to an improved model performance. A striking example of compensating model errors is described in Palmer and Weisheimer ${ }^{119}$, who report how an inadequate representation of horizontal baroclinic fluxes resulted in a model error that was equal and opposite to the systematic error caused by insufficiently represented vertical orographic gravity wave fluxes. Improvements to wave drag parameterization without increasing resolution unbalanced the compensating model errors, leading to an increase in systematic model bias.

Williams ${ }^{175}$ studied the effect of including a stochastic term in the fluxes between the atmospheric and oceanic components in a coupled ocean-atmosphere model. He reports changes to the time-mean climate and increased variability of the El Nino Southern Oscillation, suggesting that the lack of representing of sub-grid variability in air-sea fluxes may contribute to some of the biases exhibited by contemporary climate models.

On seasonal timescales where sufficient observational data for a probabilistic verification exist, stochastic parameterizations have been reported to increase predictive skill. For example, ensemble forecasts of the sea surface temperatures over the Nino3.4 region showed increased anomaly correlation, decreased bias and decreased root mean square error in coupled ocean-atmosphere models ${ }^{4,28,165}$.

\section{Conclusion}

We postulate the use of stochastic-dynamical models for uncertainty assessment and model-error representation in comprehensive Earth-System models. This need arises since even state-of-the-art weather and climate models cannot resolve all necessary processes and scales. Here we reviewed mathematical methods for stochastic climate modeling as well as stochastic subgrid-scale parameterizations and postulate their use for a more systematic strategy of parameterizing unresolved and unrepresented processes.

In the last decade, a number of studies emerged that demonstrate the potential of this approach, albeit 
applied in an ad hoc manner and tuned to specific applications. Stochastic parameterizations have been shown to provide more skillful weather forecasts than traditional ensemble prediction methods, at least on timescales where verification data exists. In addition, they have been shown to reduce longstanding climate biases, which play an important role especially for climate and climate change predictions.

Here we argue, that rather than pushing out the limit of skillful ensemble predictions by a few days, more attention should be given on the assessment of uncertainty (as already proposed, e.g., Smith ${ }^{149}$ ). Ideally, it should be carried out alongside the physical parameterization and dynamical core development and not added a posteriori. The uncertainty should be directly estimated from within the parameterization schemes and not tuned to yield a particular model performance, as is current practice. For example, Sapsis and Majda ${ }^{139}{ }^{140}$ propose a statistical framework which systematically quantifies uncertainties in a stochastic fashion.

The fact that according to the last two assessment reports (AR) of the IPCC (AR4 ${ }^{147}$ and AR5 ${ }^{150}$ ) the uncertainty in climate predictions and projections has not decreased may be a sign that we might be reaching the limit of climate predictability, which is the result of the intrinsically nonlinear character of the climate system (as first suggested by Lorenz ${ }^{82}$ ).

Recently Palmer ${ }^{121}$ argued that due to limited computational and energy power resources, predictable scales should be solved accurately, while the unpredictable scales could be represented inaccurately. This strategy is at the core of the systematic mode reduction reviewed here, but has only recently been considered for comprehensive Earth-System Models. Stochastic models focus on the accurate simulation of the large, predictable, scales, while only the statistical properties of the small, unpredictable, scales are captured. This has been demonstrated, e.g, by Franzke and Majda ${ }^{38}$, Kravtsov et al. ${ }^{74}$, who successfully applied mode reduction strategies to global atmospheric circulation models. They showed that these reduced models consisting of only 10-15 degrees of freedom reproduced many of the important statistics of the numerical circulation models which contained a few hundreds degrees of freedom. Vanden-Eijnden ${ }^{163}$ proposed numerical approaches for multi-scale systems where only the largest scales are explicitly computed and the smaller scales are approximated on the fly.

The recent result of Wouters and Lucarini ${ }^{177,178}$ provide a promising path towards a general theory of parametrizations for weather and climate models, and give theoretical support that parameterization schemes should include deterministic, stochastic and non-Markovian (memory) components. Moreover, Wouters and Lucarini's results suggest that there is common ground in developing parameterizations for weather and climate prediction models. Optimal representations of the reduced dynamics based on Ruelle's response theory and the Mori-Zwanzig formalism coincide, thus, providing equal optimal representations of the long-term statistical properties and the finite-time evolution of the slow variables.

One exciting future research area is the use of stochastic methods for use in data assimilation, which is already an active field of research ${ }^{51,54,65,104,114,115,133}$. Stochastic methods have been shown to increase the ensemble spread in data assimilation, leading to a better match between observations and model forecasts ${ }^{54,105,133}$. A cutting-edge frontier is the use of order moments and memory effects in Kalman filter data assimilation schemes ${ }^{115}$. Another emerging field is the use of stochastic parameterizations in large climate ensembles, which would allow the comparison of uncertainty estimates based in multi-models to that of stochastically perturbed ones.

Our hope is that basing stochastic-dynamic prediction on sound mathematical and statistical physics concepts will lead to substantial improvements, not only in our ability to accurately simulate weather and climate, but even more importantly to give proper estimates on the uncertainty of these predictions.

\section{Acknowledgments:}

CLEF is supported by the German Research Foundation (DFG) through the cluster of Excellence CliSAP, TJO is an Australian Research Council Future Fellow, PDW acknowledges a University Research Fellowship from the Royal Society (UF080256) and VL funding from the European Research Council (NAMASTE). 


\section{References}

[1] Achatz, U. and G. Branstator, 1999: A two-layer model with empirical linear corrections and reduced order for studies of internal climate variability. J. Atmos. Sci., 56, 3140-3160.

[2] Achatz, U. and J. D. Opsteegh, 2003: Primitive-equation-based low-order models with seasonal cycle. Part I: Model construction. J. Atmos. Sci., 60, 465-477.

[3] Berner, J., 2005: Linking nonlinearity and non-Gaussianity of planetary wave behavior by the Fokker-Planck equation. J. Atmos. Sci., 62, 2098-2117.

[4] Berner, J., F. J. Doblas-Reyes, T. N. Palmer, G. Shutts and A. Weisheimer, 2008: Impact of a quasi-stochastic cellular automaton backscatter scheme on the systematic error and seasonal prediction skill of a global climate model. Phil. Trans. R. Soc. A, 366, 2559-2577.

[5] Berner, J., G. Shutts, M. Leutbecher, and T. Palmer, 2009: A spectral stochastic kinetic energy backscatter scheme and its impact on flow-dependent predictability in the ECMWF ensemble prediction system. J. Atmos. Sci., 66, 603-626.

[6] Berner, J., S.-Y. Ha, J. P. Hacker, A. Fournier, and C. Snyder, 2011: Model uncertainty in a mesoscale ensemble prediction system: Stochastic versus multi-physics representations. Mon. Wea. Rev., 139, 1972-1995.

[7] Berner, J., T. Jung and T. N. Palmer, 2012: Systematic model error: The impact of increased horizontal resolution versus improved stochastic and deterministic parameterizations. J. Climate, 25, 4946-4962.

[8] Berner, J., K. R. Smith, S.-Y. Ha, J. P. Hacker, and C. Snyder, 2014: Increasing the skill of probabilistic forecasts: Model-error representations versus calibration and debiasing. Mon. Wea. Rev., submitted.

[9] Birner, T. and P. D. Williams, 2008: Sudden Stratospheric Warmings as Noise-Induced Transitions. J. Atmos. Sci., 65, 3337-3343.

[10] Bowler, N. E., A. Arribas, S. E. Beare, K. R. Mylne, and G. J. Shutts, 2009: The local ETKF and SKEB: Upgrades to the MOGREPS short-range ensemble prediction system. Quart. J. Roy. Meteor. Soc., 135, 767-776.

[11] Bowler, N. E., A. Arribas, K. R. Mylne, K. B. Robertson, and S. E. Beare, 2008: The MOGREPS short-range ensemble prediction system. Quart. J. Roy. Meteor. Soc., 134, 703-722.

[12] Bouttier, F., B. Vié, O. Nuissier, and L. Raynaud, 2012: Impact of stochastic physics in a convection-permitting ensemble. Monthly Weather Review, 140, 3706-3721.

[13] Buizza, R., M. Miller and T. N. Palmer, 1999: Stochastic representation of model uncertainties in the ECMWF ensemble prediction system. Q. J. R. Meteorol. Soc., 125, 2887-2908.

[14] Buizza, R., P. L. Houtekamer, Z. Toth, G. Pellerini, M. Wei and Y. Zhu, 2005: A comparison of the ECMWF, MSC and NCEP global ensemble prediction systems. Mon. Wea. Rev, 133, 1076-1097.

[15] Carnevale G. F. and J. S. Frederiksen, 1983: Viscosity renormalization based on direct-interaction closure. J. Fluid Mech., 131, 289.

[16] Charron, M., G. Pellerin, L. Spacek, P. L. Houtekamer, N. Gagnon, H. L. Mitchell, and L. Michelin, 2010: Toward random sampling of model error in the canadian ensemble prediction system. Mon. Wea. Rev., 138, $1877-1901$.

[17] Chekroun, M., H. Liu and S. Wang, 2013: On stochastic parameterizing manifolds: Pullback characterization and non-Markovian reduced equations. arxiv:1310.3896

[18] Chorin, A. J., O. H. Hald and R. Kupferman, 2002: Optimal prediction with memory. Physica D, 166, $239-257$.

[19] Chorin, A. J. and O. H. Hald, 2013: Stochastic tools in mathematics and science. Springer, New York, $208 p$ p. 
[20] Cohen, B. G. and G. C. Craig, 2005: Fluctuations in a equilibrium convective ensemble. Part II: Numerical experiments. J. Atmos. Sci., 63, 2005-2015.

[21] Crommelin, D. and E. Vanden-Eijnden, 2006: Reconstruction of diffusions using spectral data from timeseries. Comm. Math. Sci., 4, 651-668.

[22] Crommelin, D. and E. Vanden-Eijnden, 2008: Subgrid-scale parameterization with conditional Markov chains. J. Atmos. Sci., 65, 2661-2675.

[23] Culina, J., S. Kravtsov and A. H. Monahan, 2011: Stochastic parameterization schemes for use in realistic climate models. J. Atmos. Sci., 68, 284-299.

[24] Dawson, A., T. N. Palmer and S. Corti, 2012: Simulating regimes structures in weather and climate prediction models. Geophys. Res. Lett., 39, L21805.

[25] Deker U. and F. Haake, 1957: Fluctuation-dissipation theorems for classical processes. Phys. Rev. A, 6, 2043.

[26] DelSole, T. and A. Y. Hou, 1999: Empirical stochastic models for the dominant climate statistics of a General Circulation Model. J. Atmos. Sci., 56, 3436-3456.

[27] DelSole, T., 2004: Stochastic models of quasigeostrophic turbulence. Surveys Geophys., 25, 107-149.

[28] Doblas-Reyes, F., A. Weisheimer, M. Déqué, N. Keenlyside, M. McVean, J.M. Murphy, P. Rogel, D. Smith, T.N. Palmer, 2009: Addressing model uncertainty in seasonal and annual dynamical seasonal forecasts. Quart. J. Roy. Meteor. Soc., 135, 1538-1559.

[29] Dolaptchiev, S. U. Achatz and I. Timofeyev, 2013: Stochastic closure for local averages in the finite-difference discretization of the forced Burgers equation. Theor. Comput. Fluid Dyn., 27, 297-317.

[30] Douglas, J. F. and J. M. K. Gasiorek, 2000: Fluid Mechanics. Pearsons Higher Education.

[31] Eckel, F. A. and C. F. Mass, 2005: Aspects of effective mesoscale, short-range ensemble forecasting. Wea. For., $20,328-350$.

[32] Epstein, E. S., 1969: Stochastic dynamic prediction. Tellus, 21, 739-759.

[33] Epstein, E. S. and Pitcher E. J., 1972: Stochastic analysis of meteorological fields. J. Atmos. Sci., 29, $244-257$.

[34] Farrell, B. F. and P. J. Ioannou, 1993: Stochastic Dynamics of baroclinic waves. J. Atmos. Sci., 50, $4044-4057$.

[35] Farrell, B. F. and P. J. Ioannou, 1995: Stochastic Dynamics of the midlatitude atmospheric jet. J. Atmos. Sci., $52,1642-1656$.

[36] Frankignoul, C. and K. Hasselmann, 1977: Stochastic climate models, Part II: Application to sea-surface temperature anomalies and thermocline variability. Tellus, 29, 289-305.

[37] Franzke, C., A. J. Majda and E. Vanden-Eijnden, 2005: Low-Order Stochastic Mode Reduction for a Realistic Barotropic Model Climate. J. Atmos. Sci., 62, 1722-1745.

[38] Franzke C. and A. J. Majda, 2006: Low-Order Stochastic Mode Reduction for a Prototype Atmospheric GCM. J. Atmos. Sci., 63, 457-479.

[39] Franzke, C., A. J. Majda and G. Branstator, 2007: The origin of nonlinear signatures of Planetary Wave Dynamics: Mean Phase Space Tendencies and Contributions from Non-Gaussianity. J. Atmos. Sci., 64, 3987-4003. doi: 10.1175/2006JAS2221.1

[40] Franzke, C., 2012: Predictability of extreme events in a nonlinear stochastic-dynamical model. Phys. Rev. E, 85, doi: 10.1103/PhysRevE.85.031134 
[41] Franzke, C., 2013: Predictions of critical transitions with non-stationary reduced order models. Physica D, 262, 35-47.

[42] Frederiksen J. S., 1999: Subgrid-scale parameterizations of eddy-topographic force, eddy viscosity, and stochastic backscatter for flow over topography. J. Atmos. Sci., 56, 1481.

[43] Frederiksen J. S. and A. G. Davies, 1997: Eddy viscosity and stochastic backscatter parameterizations on the sphere for atmospheric circulation models. J. Atmos. Sci., 54, 2475.

[44] Frederiksen J.S., A. G. Davies and R. C. Bell, 1994: Closure equations with non-Gaussian restarts for truncated two-dimensional turbulence. Phys. Fluids, 6, 3153.

[45] Frederiksen J. S. and A. G. Davies, 2000: Dynamics and spectra of cumulant update closures for two-dimensional turbulence. Geophys. Astrophys. Fluid Dynam., 92, 197.

[46] Frederiksen J. S. and T. J. O'Kane, 2005: Inhomogeneous closure and statistical mechanics for Rossby wave turbulence over topography. J. Fluid Mech., 539, 137.

[47] Frenkel, Y., A. J. Majda and B. Khouider, 2011: Using the stochastic multicloud model to improve tropical convective parameterizations: A paradigm example. J. Atmos. Sci., 69, 1080-1105.

[48] Gallavotti, G. and E. G. D. Cohen, 1995: Dynamical ensembles in stationary states. J. Stat. Phys., 80, $931-970$.

[49] Gardiner, C. W., Handbook of stochastic methods, 1985. Springer-Verlag, 442pp.

[50] Givon, D., P. Kupferman and A. Stuart, 2004: Extracting macroscopic dynamics: model problems and algorithms. Nonlinearity, 17, R55.

[51] Gottwald, G. and J. Harlim, 2013: The role of additive and multiplicative moise in filtering complex dynamical systems. Proc. Roy. Soc. A, 469, 20130096.

[52] Gottwald, G. and I. Melbourne, 2013: Homogenization for deterministic maps and multiplicative noise. Proc. Roy. Soc. A, 469, 20130201.

[53] Jung, T., T. N. Palmer, and G. J. Shutts, 2005: Influence of a stochastic parameterization on the frequency of occurrence of north pacific weather regimes in the ECMWF model. Geophys. Res. Lett., 32, L23811.

[54] Ha, S.-Y., J. Berner, and C. Snyder, 2014: Model error representation in mesoscale wrf-dart cycling. to be submitted to Mon. Wea. Rev..

[55] Hagedorn, R., F. J. Doblas-Reyes, and T. N. Palmer, 2005: The rationale behind the success of multi-model ensembles in seasonal forecasting - i. basic concept. Tellus, 57A, 219-233.

[56] Hasselmann, K., Stochastic Climate Models: Part I. Theory. Tellus, 28 (1976), 473-484.

[57] Harlim, J., A. Mahdi and A. J. Majda, 2013: An ensemble Kalman filter for statistical estimation of physics constrained nonlinear regression models. J. Compt. Phys., 257, 782-812.

[58] Heinz, S., 2003: Statistical mechanics of turbulent flows. Springer, Berlin, 215pp.

[59] Herring J. R., 1965: Self-consistent-field approach to turbulence theory. Phys. Fluids, 8, 2219.

[60] Herring J.R. and Kraichnan R.H., 1972: Statistical Models of Turbulence. Springer, 1972.

[61] Hide, R., P. J. Mason and R. A. Plumb, 1977: Thermal convection in a rotating fluid subject to a horizontal temperature gradient: spatial and temporal characteristics of fully developed baroclinic waves. J. Atmos. Sci., 34, 930-950.

[62] Holloway G., 1992: Representing topographic stress for large-scale ocean models. J. Phys. Oceanogr., $22,1033$. 
[63] Houtekamer, P. L., L. Lefaivre, J. Derome, H. Ritchie, and H. L. Mitchell, 1996: A system simulation approach to ensemble prediction. Mon. Wea. Rev., 124, 1225-1242.

[64] Imkeller, P. and J.-S. von Storch, 2001: Stochastic Climate Models. Birkhäuser, 398 pages.

[65] Isaksen, L., M. Fisher, and J. Berner, 2007: Use of analysis ensembles in estimating flow-dependent background error variances. Proceedings of ECMWF Workshop on Flow Dependent Background Errors ECMWF, June 11-13, 2007, Reading, United Kingdom, 65-86 pp.

[66] Khasminsky, R. Z., 1963: Principle of averaging for parabolic and elliptic differential equations and for Markov processes with small diffusions. Theory Probab. Appl., 8, 1-21.

[67] Khouider, B., J. Biello and A. J. Majda, 2010: A stochastic multicloud model for tropical convection. Commun. Math. Sci., 8, 187-216.

[68] Kitsios V., J. S. Frederiksen and M. J. Zidikheri, 2012: Subgrid model with scaling laws for atmospheric flows. J. Atmos. Sci., 69, 1427.

[69] Klein, R., 2010: Scale-dependent models for atmospheric flows. Ann. Rev. Fluid Mech., 42, 249-274.

[70] Kondrashov, D., S. Kravtsov and M. Ghil, 2006: Empirical mode reduction in a model of extratropical lowfrequency variability. J. Atmos. Sci., 63, 1859-1877.

[71] Kraichnan R. H., 1959: The structure of isotropic turbulence at very high reynolds number. J. Fluid. Mech., 5, 497.

[72] Kraichnan R. H., 1972: Test-field model for inhomogeneous turbulence. J. Fluid Mech., 56, 287.

[73] Kraichnan R. H., 1976: Eddy viscosity in two and three dimensions. J. Atmos. Sci., 33, 1521.

[74] Kravtsov, S., D. Kondrashov and M. Ghil, 2005: Multilevel regression modeling of nonlinear processes: derivation and applications to climatic variability. J. Climate, 18, 4404-4424.

[75] Krishnamurti, T. N., C. M. Kishtawal, Z. Zhang, T. LaRow, D. Bachiochi, E. Williford, S. Gadgil, , and S. Surendran, 2000: Multimodel ensemble forecasts for weather and seasonal climate. J. Climate, 13, 4196-4216.

[76] Kurtz, T. G., 1973: A limit theorem for perturbed operator semi-groups with applications to random evolutions. J. Funct. Anal., 12, 55-67.

[77] Leith C. E., 1971: Atmospheric predictability and two-dimensional turbulence. J. Atmos. Sci., $28,145$.

[78] Leith C. E., Theoretical skill of Monte Carlo forecasts. Mon. Wea. Rev., 102, 409.

[79] Leith, C. E., and R. H. Kraichnan, 1972: Predictability of turbulent flows. J. Atmos. Sci., 29, 1041-1058.

[80] Leslie D.C. and G. L. Quarini, 1979: The application of turbulence theory to the formulation of subgrid modelling procedures. J. Fluid Mech., 91, 65.

[81] Lin, J. W. B. and J. D. Neelin, 2000: Influence of a stochastic moist convective parameterization on tropical climate variability. Geophys. Res. Lett., 27, 3691-3694.

[82] Lorenz, E. N., 1963: Deterministic nonperiodic flow. J. Atmos. Sci., 20, 130-141.

[83] Lorenz, E. N., 1965: A study of the predictability of a 28-variable atmospheric model. Tellus, 17, 321-333.

[84] Lorenz, E. N., 1969: Atmospheric predictability as revealed by naturally occcuring analogues. J. Atmos. Sci., 26, 636-646.

[85] Lovegrove, A. F., P. L. Read and C. J. Richards, 2000: Generation of inertia-gravity waves in a baroclinically unstable fluid. Q. J. Roy. Meteorol. Soc., 126, 3233-3254. 
[86] Lemons, D. S., 2002: An introduction to stochastic processes in physics. Johns Hopkins University Press, 115pp.

[87] Lucarini, V., 2009: Evidence for dispersion relations for the nonlinear response of the Lorenz 1963 system. J. Stat. Phys., 134, 381-400.

[88] Lucarini, V. and S. Sarno 2011: A statistical mechanic approach for the computation of the claimatic response to general forcings. Nonlin. Proc. Geophys., 18, 7-28.

[89] Majda, A. J., I. Timofeyev and E. Vanden-Eijnden, 1999: Models for stochastic climate prediction. Proc. Nat. Acad. Sci. USA, 96, 14687-14691.

[90] Majda, A. J., I. Timofeyev and E. Vanden-Eijnden, 2001: A mathematical framework for stochastic climate models. Commun. Pure Appl. Math., 54, 891-974.

[91] Majda, A. J., I. Timofeyev and E. Vanden-Eijnden, 2002: A priori tests of a stochastic mode reduction strategy. Physica D, 170, 206-252.

[92] Majda, A. J., I. Timofeyev and E. Vanden-Eijnden, 2003: Systematic strategies for stochastic mode reduction in climate. J. Atmos. Sci., 60, 1705-1722.

[93] Majda, A. J., R. Abramov and M. Grote, 2005: Information Theory and Stochastics for Multiscale Nonlinear Systems. CRM Monograph Series, American Mathematical Society.

[94] Majda, A. J., C. Franzke and B. Khouider, 2008: An applied mathematics perspective on stochastic modelling for climate. Phil. Trans. R. Soc. A, 366, 2429-2455.

[95] Majda, A. J. and S. Stechmann, 2008: Stochastic models for convective momentum transport. Proc. Nat. Acad. USA, 105, 17614-17619.

[96] Majda, A. J., C. Franzke and D. Crommelin, 2009: Normal forms for reduced stochastic climate models. Proc. Nat. Acad. Sci. USA, 106, 3649-3653. doi: 10.1073/pnas.0900173106

[97] Majda, A. J. and J. Harlim, 2013: Physics constrained nonlinear regression models for time series. Nonlinearity, $26,201-217$.

[98] Mason, P. and D. Thomson, 1992: Stochastic backscatter in large-eddy simulations of boundary layers. J. Fluid Mech., 242, 51-78.

[99] McComb W. D., 1974: A local energy-transfer theory of isotropic turbulence. J. Phys. A, 7, 632.

[100] McComb W. D. A. Hunte and C. Johnson, 2001a: Conditional mode elimination and the subgrid-modelling problem for isotropic turbulence. Phys. Fluids, 13, 2030.

[101] Melbourne, I. and A. M. Stuart, 2011: A note on diffusion limits of chaotic skew-product flows. Nonlinearity, 24, 1361-1367.

[102] Métais O. and M. Lesieur, 1986: Statistical predictability of decaying turbulence. J. Atmos. Sci., 43, 857.

[103] Monahan, A. H. and J. Culina, 2011: Stochastic averaging of idealized climate models. J. Climate, 24, 3068-3088.

[104] Miller, R. N., E. F. Carter and S. T. Blue, 1999: Data assimilation into nonlinear stochastic models. Tellus, 51A, 167-194.

[105] Mitchell, L. and G. A. Gottwald, 2012: Data assimilation in slow-fast systems using homogenized climate models. J. Atmos. Sci., 69, 1359-1377.

[106] Murphy, J. M., D. M. Sexton, D. N. Barnett, G. S. Jones, M. J. Webb, M Collins and D. A. Stainforth, 2004: Quantification of modelling uncertainties in a large ensemble of climate change simulations. Nature, 430, 768-772. 
[107] Memin, E., 2014: Fluid flow dynamics under location uncertainty. Geophys. Astrophys. Fluid Dyn., 108, 119-146.

[108] Molteni, F. and T. N. Palmer, 1993: Predictability and finite-time instability of the Northern winter circulation. Quart. J. Roy. Meteor. Soc., 119, 269-298.

[109] Ogura Y., 1963: A consequence of the zero fourth cumulant approximation in the decay of isotropic turbulence. J. Fluid Mech., 16, 33.

[110] O'Kane T. J., R. E. Scholten, M. R. Walkiewwicz and P. M. Farrell, 1999: Nonlinear interactions of multi-level atoms with a near resonant standing wave. Phys. Rev. A, 59, 4485-4493.

[111] O'Kane T. J. and J. S. Frederiksen, 2004: The QDIA and regularized QDIA closures for inhomogeneous turbulence over topography. J. Fluid Mech., 504, 133.

[112] O'Kane T. J. and J. S. Frederiksen, 2008a: Statistical dynamical subgrid-scale parameterizations for geophysical flows. Phys. Scr., T132, 014033.

[113] O'Kane T. J. and J. S. Frederiksen, 2008b: A comparison of statistical dynamical and ensemble prediction methods during blocking. J. Atmos. Sci., 65, 426.

[114] O'Kane T. J. and J. S. Frederiksen, 2008c: Comparison of statistical dynamical, square root and ensemble Kalman filters. Entropy, 10, 684-721. DOI: 10.3390/e10040684.

[115] O'Kane T. J. and J. S. Frederiksen, 2010: The application of statistical dynamical turbulence closures to data assimilation. Phys. Scr., T142, 014042.

[116] Orszag S. A., 1970: Analytical theories of turbulence. J. Fluid Mech. 41, 363.

[117] Palmer, T. N., 1999: A nonlinear dynamical perspective on climate prediction. J. Climate, 12, 575-591.

[118] Palmer, T. N., 2001: A nonlinear dynamical perspective on model error: A proposal for non-local stochasticdynamic parameterization in weather and climate prediction. Quart. J. Roy. Meteor. Soc., 127, 279-304.

[119] Palmer, T. N., and A. Weisheimer, 2011: Diagnosing the causes of bias in climate models - why is it so hard? Geophysical \& Astrophysical Fluid Dynamics, 105, 351-365.

[120] Palmer, T. N. and P. D. Williams, 2008: Introduction. Stochastic physics and climate modelling. Phil. Trans. Roy. Soc. A, 366(1875), 2421-2427.

[121] Palmer, T., 2014: More reliable forecasts with less precise computations: a fast-track route to cloud-resolved weather and climate simulators? Phil. Trans. A, 372, 20130391.

[122] Palmer, T., U. Andersen, P. Cantelaube, M. Davey, M. Deque, F. Doblas-Reyes, H. Feddersen, R. Graham, S. Gualdi, J.-F. Gueremy, et al., 2004: Development of a European multi-model ensemble system for seasonal to inter-annual prediction (DEMETER). Bulletin of the American Meteorological Society, 85, 853-872.

[123] Palmer, T. N., R. Buizza, F. Doblas-Reyes, T. Jung, M. Leutbecher, G. Shutts, M. Steinheimer, and A. Weisheimer, 2009: Stochastic Parametrization and Model Uncertainty. ECMWF Technical Memorandum, 598, available at http://www.ecmwf.int/publications/.

[124] Papanicolaou, G. C., 1976: Some probabilistic problems and methods in singular perturbations. Rocky Mountain J. Math., 6, 653-674.

[125] Pavliotis, G. A. and A. M. Stuart, 2008: Multiscale Methods: Averaging and Homogenization. Springer, 307pp.

[126] Peavoy, D., C. Franzke and G. Roberts, 2014: Systematic Physics Constrained Parameter Estimation of Stochastic Differential Equations. Comp. Stat. Data Analys., accepted pending minor revisions. 
[127] Penland, C. and L. Matrosova, 1994: A balance condition for stochastic numerical models with application to the El Nino-Southern Oscillation. J. Climate., 7, 1352-1372.

[128] Penland, C. and P. D. Sardeshmukh, 1995: The optimal growth of tropical sea surface temperature anomalies. J. Climate., 8, 1999-2014.

[129] Penland, C., 2003: Noise out of chaos and why it won't go away. Bull. Amet. Meteorol. Soc., 84, 921-925.

[130] Pitcher, E. J., 1977: Application of stochastic dynamic prediction to real data. J. Atmos. Sci., 34, 3-21. lmer

[131] Plant, R. S. and G. C. Craig, 2008: A stochastic parameterization for deep convection based on equillibrium statistics. J. Atmos. Sci., 65, 87-105.

[132] Reynolds, C. A., J. G. McLay, J. S. Goerss, E. A. Serra, D. Hodyss and C. R. Sampson, 2011: Impact of resolution and design on the U.S. Navy global ensemble performance in the tropics. Mon. Wea. Rev., 139, 2145-2155.

[133] Romine, G. S., C. S. Schwartz, J. Berner, K. R. Smith, C. Snyder, J. L. Anderson, and M. L. Weisman, 2014: Representing forecast error in a convection-permitting ensemble system. Mon. Wea. Rev., submitted.

[134] Rose H. A., 1977: Eddy diffusivity, eddy noise and subgrid-scale modelling. J. Fluid Mech., 81, 719.

[135] Rose H. A., 1985: An efficient non-Markovian theory of non-equilibrium dynamics. Physica D, $14,216$.

[136] Ruelle, D., 1999: Smooth dynamics and new theoretical ideas from nonequilibrium statistical mechanics. J. Stat. Phys., 95, 393-468.

[137] Ruelle, D., 2009: A review of linear response theory for general differentiable dynamical systems. Nonlinearity, 22, 855 .

[138] Sanchez, C., K. D. Williams, G. Shutts, and M. Collins, 2014: Impact of a stochastic kinetic energy backscatter scheme across time-scales and resolutions. Q.J.R. Meteorol. Soc., doi: 10.1002/qj.2328, 577-599.

[139] Sapsis, T. and A. J. Majda, 2013a: A statistically accurate modified quasilinear Gaussian closure for uncertainty quantification in turbulent dynamical systems. Physica D, 252, 34-45.

[140] Sapsis, T. and A. J. Majda, 2013b: Statistically accurate low-order models for uncertainty quantification in turbulent dynamical systems. Proc. Nat. Acad. USA, 110, 13705-13710.

[141] Sardeshmukh, P., C. Penland and M. Newman, 2001: Rossby waves in a stochastically fluctuating medium. Stochastic Climate Models, Birkhäuser Basel, 396-384.

[142] Shutts, G. J., 2005: A kinetic energy backscatter algorithm for use in ensemble prediction systems. Quart. J. Roy. Meteor. Soc., 612, 3079-3102.

[143] Shutts, G. J. and T. N. Palmer, 2003: The use of high resolution numerical simulations of tropical circulation to calibrate stochastic physics schemes. ECMWF/CLIVAR workshop on simulation and prediction of intra-seasonal variability with the emphasis on the MJO, ECMWF, available from http://www.ecmwf.int/publications, 83-102.

[144] Shutts, G. J., and T. N. Palmer, 2007: Convective Forcing Fluctuations in a Cloud-Resolving Model: Relevance to the Stochastic Parameterization Problem. J. Climate, 20, 187-202.

[145] Shutts, G., T. Allen, and J. Berner, 2008: Stochastic parametrization of multiscale processes using a dual-grid approach. Philosophical Transactions of the Royal Society A: Mathematical, Physical and Engineering Sciences, 366, 2623-2639.

[146] Siegert, S., R. Friedrich and J. Peinke, 1998: Analysis of data sets of stochastic systems. Phys. Lett. A, 243, $275-280$. 
[147] Solomon, S., E., D. Qin, M. Manning, Z. Chen, M. Marquis, K. Averyt, M. Tignor, and H. Miller, 2007: Climate Change 2007: The Physical Science Basis. Cambridge University Press, New York, 996pp pp.

[148] Smagorinsky J., 1963: General circulation experiments with the primitive equations. I. The basic experiment. Mon. Wea. Rev., 91, 99.

[149] Smith, L. A., 2001: Disentangling uncertainty and error: On the predictability of nonlinear systems. Springer.

[150] Stocker, T. F., D. Qin, G.-K. Plattner, M. Tignor, S.K. Allen, J. Boschung, A. Nauels, Y. Xia, V. Bex and P.M. Midgley, 2013: Climate Change 2013: The Physical Science Basis. Cambridge University Press, Cambridge, 1535 pp.

[151] Stainforth, D., T. Aina , C. Christensen, M. Collins , N. Faull , D. J. Frame, J. A. Kettleborough , S. Knight , A. Martin , J. M. Murphy , C. Piani, D. Sexton, L. A. Smith , R. A. Spicer , A. J. Thorpe and M. R. Allen, 2005: Uncertainty in predictions of the climate response to rising levels of greenhouse gases. Nature, 433, 403-406.

[152] Stensrud, D. J., J.-W. Bao and T. T. Warner, 2000: Using initial condition and model physics perturbations in short-range ensemble simulations of mesoscale convective systems. Mon. Wea. Rev., 128, 2077-2107.

[153] Stensrud, D. J., 2007: Parameterization Schemes: Keys to understanding Numerical Weather prediction models. Cambridge University Press, Cambridge, 459pp.

[154] Stinis, P., 2006: A comparative study of two stochastic mode reduction methods. Physica D, 213, 197-213.

[155] Sura, P., 2011: A general perspective of extreme events in weather and climate. Atmos. Res., 101, 1-21.

[156] Sura, P., 2013: Stochastic models of climate extremes: Theory and observations. In Extremes in a changing world: detection, analysis and uncertainty. Eds.: AghaKouchak et al., Springer, 432pp.

[157] Stocker, E. T. F., D. Qin, G.-K. Plattner, M. Tignor, S. Allen, J. Boschung, A. Nauels, Y. Xia, V. Bex, and P. Midgley, 2013: Climate Change 2013: The Physical Science Basis. Cambridge University Press, Cambridge, United Kingdom and New York.

[158] De Swart, H. E. and J. Grasman, 1987: Effect of stochastic perturbations on a low-order spectral model of the atmospheric circulation. Tellus A, 39, 10-24.

[159] Teixera, J. and C. A. Reynolds, 2008: Stochastic nature of physical parameterization in ensemble prediction: a stochastic convection approach. Mon. Wea. Rev., 136, 483-496.

[160] Tian, Y., E. R. Weeks, K. Ide, J. S. Urbach, C. N. Baroud, M. Ghil and H. L. Swinney, 2001: Experimental and numerical studies of an eastward jet over topography. J. Fluid Mech., 438, 129-157.

[161] Toth, Z. and E. Kalnay, 1993: Ensemble forecasting at nmc: The generation of perturbations. Bull. Amer. Meteor. Soc., 74, 2317-2330.

[162] Tribbia, J. J. and D. P. Baumhefner, 2004: Scale interactions and atmospheric predictability: an updated perspective. Mon. Wea. Rev., 132, 703-713.

[163] Vanden-Eijnden, E., 2003: Numerical techniques for multi-scale dynamical systems with stochastic effects. Comm. Math. Sci., 1, 385-391.

[164] Weeks, E. R., Y. Tian, J. S. Urbach, K. Ide, H. L. Swinney and M. Ghil, 1997: Transitions Between Blocked and Zonal Flows in a Rotating Annulus with Topography. Science, 278, 1598-1601.

[165] Weisheimer, A., T. Palmer, and F. Doblas-Reyes, 2011: Assessment of representations of model uncertainty in monthly and seasonal forecast ensembles. Geophysical Research Letters, $\mathbf{3 8}$.

[166] Whitaker, J. S. and P. D. Sardeshmukh, 1998: A linear theory of extratropical synoptic eddy statistics. J. Atmos. Sci., 55, 237-258. 
[167] Wilks, D. S., 2005: Effects of stochastic parameterizations in the Lorenz'96 system. Q. J. R. Meteorol. Soc., 131, 389-407.

[168] Williams, P. D., P. L. Read and T. W. N. Haine, 2003: Spontaneous generation and impact of inertia-gravity waves in a stratified, two-layer shear flow. Geophys. Res. Lett., 30, 2255.

[169] Williams, P. D., T. W. N. Haine and P. L. Read, 2004a: Stochastic resonance in a nonlinear model of a rotating, stratified shear flow, with a simple stochastic inertia-gravity wave parameterization. Nonlinear. Proc. Geophys., $11,127-135$.

[170] Williams, P. D., P. L. Read and T. W. N. Haine, 2004b: A calibrated, non-invasive method for measuring the internal interface height field at high resolution in the rotating, two-layer annulus. Geophys. Astrophys. Fluid Dyn., 6, 453-471.

[171] Williams, P. D., 2005: Modelling climate change: the role of unresolved processes. Phil. Trans. Roy. Soc. A, 363(1837), 2931-2946.

[172] Williams, P. D., T. W. N. Haine and P. L. Read, 2005: On the generation mechanisms of short-scale unbalanced modes in rotating two-layer flows with vertical shear. J. Fluid Mech., 528, 1-22.

[173] Williams, P. D., T. W. N. Haine and P. L. Read, 2008: Inertia-gravity waves emitted from balanced flow: Observations, properties, and consequences. J. Atmos. Sci., 65, 3543-3556.

[174] Williams, P. D., T. W. N. Haine, P. L. Read, S. R. Lewis and Y. H. Yamazaki, 2009: QUAGMIRE v1.3: a quasi-geostrophic model for investigating rotating fluids experiments. Geoscientific Model Development, $2,13-32$.

[175] Williams, P. D., 2012: Climatic impacts of stochastic fluctuations in air-sea fluxes. Geophysical Research Letters, 39.

[176] Winkler, C. R., M. Newman and P. D. Sardeshmukh, 2001: A linear model of wintertime low-frequency variability. Part I: Formulation and forecast skill. J. Climate, 14, 4474-4494.

[177] Wouters, J. and V. Lucarini, 2012: Disentangling multi-level systems: averaging, correlations and memory. J. Stat. Mech., PO3003.

[178] Wouters, J. and V. Lucarini, 2013: Multi-level dynamical systems: Connecting the Ruelle response theory and the Mori-Zwanzig approach. J. Stat. Phys., 151, 850-860.

[179] Zhang, Y. and I. M. Held, 1999: A linear stochastic model of a GCM's midlatitude storm tracks. J. Atmos. Sci., $56,3416-3435$.

[180] Zidikheri M. J. and J. S. Frederiksen, 2009: Stochastic subgrid parameterizations for simulations of atmospheric baroclinic flows. J. Atmos. Sci., 66, 2844. 


\section{List of Figures}

1 Regime transitions in a rotating two-layer annulus laboratory experiment, viewed from above. Different colours correspond to different internal interface heights, through the use of a sophisticated visualisation technique ${ }^{170}$. In the upper row, small-scale inertia-gravity waves are absent, and large-scale regime transitions do not occur. In the lower row, small-scale inertia-gravity waves are present locally in the troughs of the large-scale wave, and a large-scale regime transition does occur. From the laboratory experiments of Williams et al. ${ }^{168,169,172,173}$.

2 Mean systematic error of $500 \mathrm{hPa}$ geopotential height fields (shading) for extended boreal winters (December-March) of the period 1962-2005. Errors are defined with regard to the observed mean field (contours), consisting of a combination of ERA-40 (1962-2001) and operational ECMWF analyses (2002-2005). (a) Systematic error in a numerical simulation with the ECMWF model IFS, version CY32R1, run at a horizontal resolution of $T_{L} 95$ (about $210 \mathrm{~km}$ ) and 91 vertical levels. (b) Systematic error in a simulation with a stochastic kinetic-energy backscatter scheme (SKEBS). Significant differences at the $95 \%$ confidence level based on a Student's t-test are hatched. After Berner et al. ${ }^{7}$. 

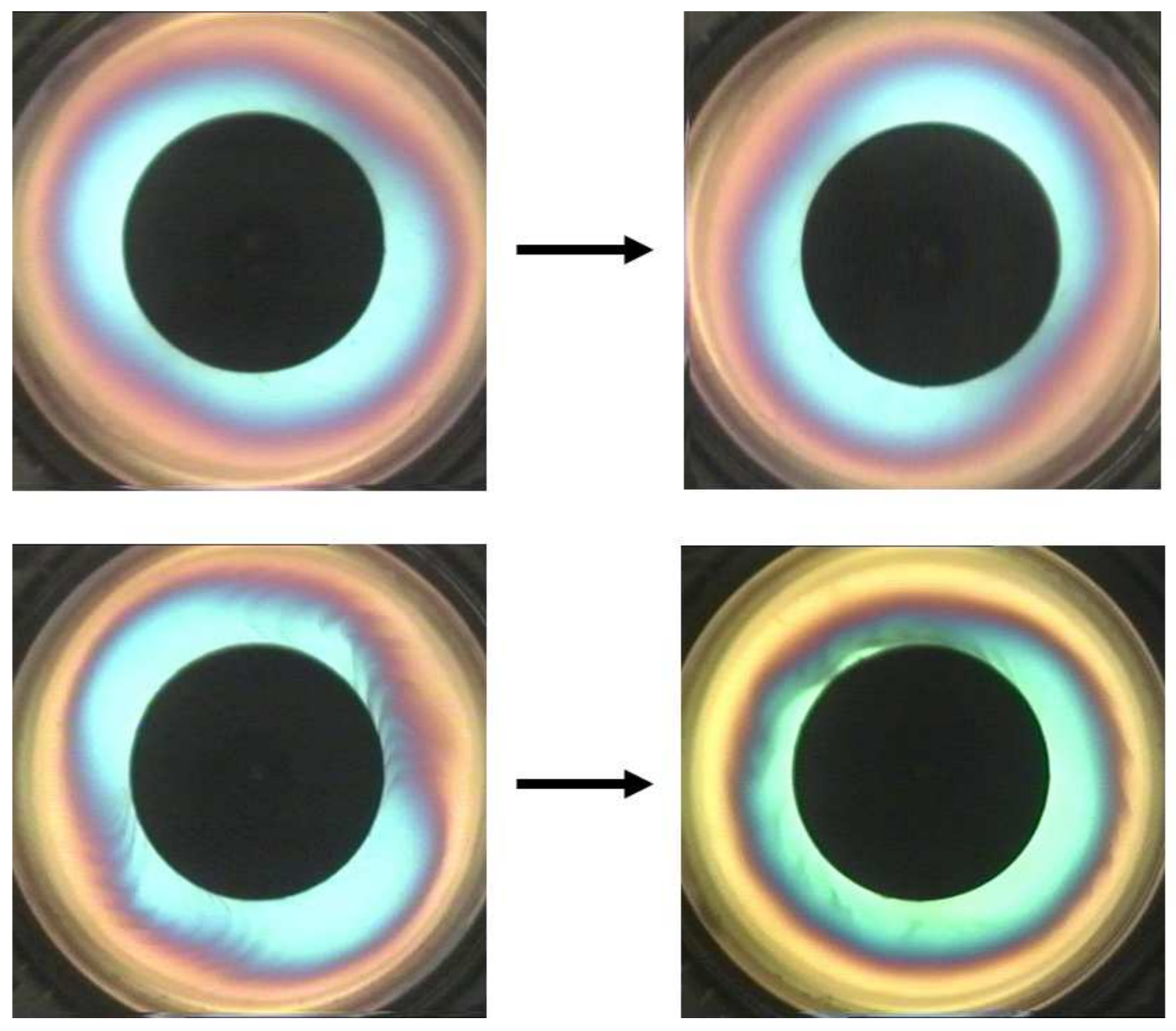

Figure 1: Regime transitions in a rotating two-layer annulus laboratory experiment, viewed from above. Different colours correspond to different internal interface heights, through the use of a sophisticated visualisation technique $^{170}$. In the upper row, small-scale inertia-gravity waves are absent, and large-scale regime transitions do not occur. In the lower row, small-scale inertia-gravity waves are present locally in the troughs of the large-scale wave, and a large-scale regime transition does occur. From the laboratory experiments of Williams et al. ${ }^{168,169,172,173}$. 
a)

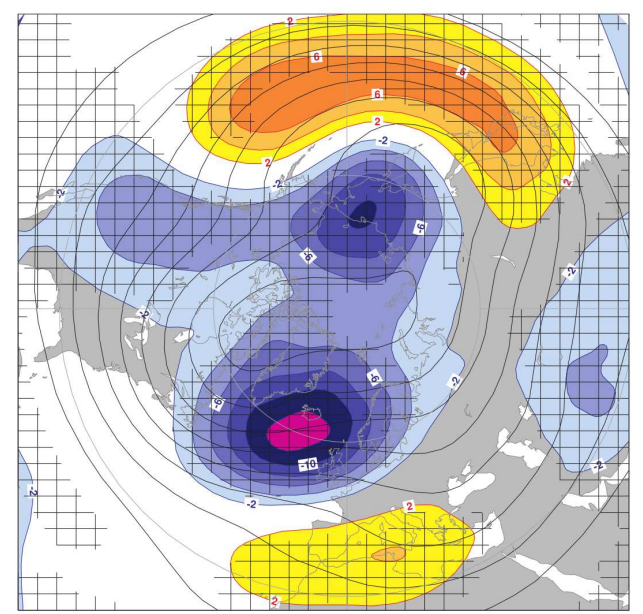

b)

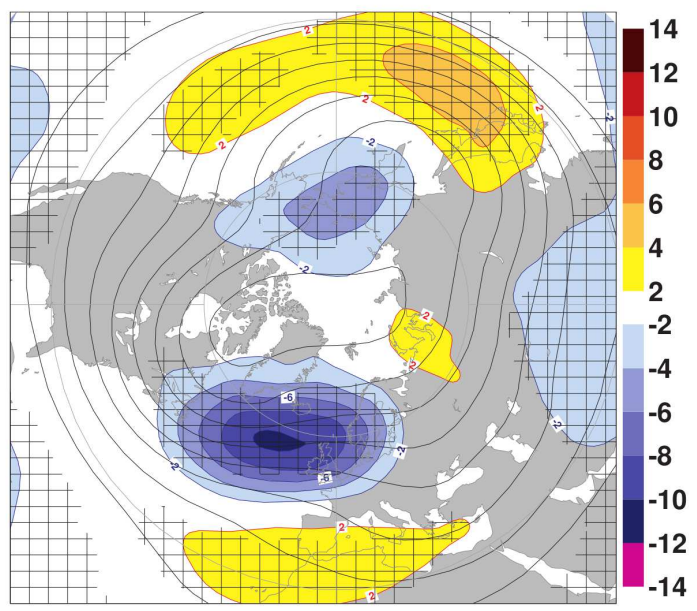

Figure 2: Mean systematic error of $500 \mathrm{hPa}$ geopotential height fields (shading) for extended boreal winters (December-March) of the period 1962-2005. Errors are defined with regard to the observed mean field (contours), consisting of a combination of ERA-40 (1962-2001) and operational ECMWF analyses (2002-2005). (a) Systematic error in a numerical simulation with the ECMWF model IFS, version CY32R1, run at a horizontal resolution of $T_{L} 95$ (about $210 \mathrm{~km}$ ) and 91 vertical levels. (b) Systematic error in a simulation with a stochastic kinetic-energy backscatter scheme (SKEBS). Significant differences at the $95 \%$ confidence level based on a Student's t-test are hatched. After Berner et al. ${ }^{7}$. 